\title{
Semi-insulating GaAs detectors with HDPE layer for detection of fast neutrons from D-T nuclear reaction
}

\author{
Andrea Sagatova \\ Institute of Nuclear and Physical Engineering, Faculty of Electrical Engineering and Information Technology, \\ Slovak University of Technology in Bratislava, Ilkovičova 3, SK-81219 Bratislava, Slovak Republic \\ andrea.sagatova@stuba.sk \\ University Centre of Electron Accelerators, Slovak Medical University, Ku kyselke 497, SK-91106 Trenčin, \\ Slovak Republic \\ asagatova@szu.sk \\ Bohumir Zatko \\ Institute of Electrical Engineering, Slovak Academy of Sciences, Dúbravská cesta 9, SK-814 04 Bratislava, \\ Slovak Republic \\ elekbzat@savba.sk \\ Katarina Sedlackova*, Marius Pavlovic ${ }^{\dagger}$ and Vladimir Necas ${ }^{\ddagger}$ \\ Institute of Nuclear and Physical Engineering, Faculty of Electrical Engineering and Information Technology, \\ Slovak University of Technology in Bratislava, Ilkovičova 3, SK-81219 Bratislava, Slovak Republic \\ *katarina.sedlackova@stuba.sk, ‘marius.pavlovic@stuba.sk, ${ }^{\dagger} v l a d i m i r . n e c a s @ s t u b a . s k$

\section{Marko Fulop} \\ University Centre of Electron Accelerators, Slovak Medical University, Ku kyselke, 497 SK-91106 Trenčin, \\ Slovak Republic \\ marko.fulop@szu.sk \\ Michael Solar ${ }^{\S}$ and Carlos Granja \\ Institute of Experimental and Applied Physics, Czech Technical University in Prague, Horská 3a/22, CZ -12800 \\ Prague 2, Czech Republic \\ §michael.solar@utef.cvut.cz, `carlos.granja@utef.cvut.cz \\ Published 1 September 2016
}

\begin{abstract}
Bulk semi-insulating (SI) GaAs detectors optimized for fast-neutron detection were examined using mono-energetic neutrons. The detectors have an active area of $7.36 \mathrm{~mm}^{2}$ defined by a multipixel structure of a AuZn Schottky contact allowing a relatively high breakdown voltage $(300 \mathrm{~V})$ sufficient for full depletion of the detector structure. The Schottky contact is covered by a HDPE (high density polyethylene) conversion layer, where neutrons transfer their kinetic energy to hydrogen atoms through elastic nuclear collisions. The detectors were exposed to mono-energetic neutrons generated by a deuterium (D)-tritium (T) nuclear reaction at a Van de Graaff accelerator.
\end{abstract}

This is an Open Access article published by World Scientific Publishing Company. It is distributed under the terms of the Creative Commons Attribution 3.0 (CC-BY) License. Further distribution of this work is permitted, provided the original work is properly cited. 


\begin{abstract}
Neutrons reached a kinetic energy of $16.8 \mathrm{MeV}$ when deuterons were accelerated by $1 \mathrm{MV}$ potential. The influence of the HDPE layer thickness on the detection efficiency of the fast neutrons was studied. The thickness of the conversion layer varied from $50 \mu \mathrm{m}$ to $1300 \mu \mathrm{m}$. The increase of the HDPE layer thickness led to a higher detection efficiency due to higher conversion efficiency of the HDPE layer. The effect of the active detector thickness modified by the detector reverse bias voltage on the detection efficiency was also evaluated. By increasing the detector reverse voltage, the detector active volume expands to the depth and also to the sides, slightly increasing the neutron detection efficiency.
\end{abstract}

Keywords: Solid-state detectors; GaAs; HDPE; detection efficiency; fast neutrons.

PACS numbers: 29.40.W, 73.30.+y, 29.25.Dz.

\title{
1. Introduction
}

Detectors of fast neutrons find applications in many areas such as nuclear medicine, nuclear power engineering, high-energy physics, materials science, and astrophysics. Applications have recently been extended to petrology, archaeology, mining, geology, and security, thanks to neutron imaging, high-speed tomography, or neutron resonance imaging techniques. Semiconductor based detectors with a neutron conversion layer present a very promising solution of fast neutron detectors, exhibiting compactness, small dimensions and high spatial resolution. Recent processing methods for silicon and gallium arsenide allow realization of moderate- to high-efficiency neutron detectors. ${ }^{1}$ Conversion layers for fast neutrons are attached to the detector in contact planar geometry and are usually based on polyethylene $(\mathrm{PE})^{2,3,4}$ and high density polyethylene (HDPE), ${ }^{5,6,7}$ but also on fast plastic scintillator. ${ }^{8}$

We have optimized our bulk semi-insulating (SI) GaAs detectors for fast-neutron detection by a multi-pixel structure of a Schottky contact. ${ }^{7}$ Neutrons are converted by $(n, p)$ elastic nuclear collisions in an HDPE layer covering the Schottky contact. In our previous research described in Ref. 7, detectors were tested using a ${ }^{239} \mathrm{Pu}$-Be source of fast neutrons with a continuous spectrum up to $12 \mathrm{MeV}$ and mean energy of about $4 \mathrm{MeV}$. The maximum obtained experimental detection efficiency was $0.113 \%$ with a $500 \mu \mathrm{m}$ thick HDPE layer at a $100 \mathrm{~V}$ reverse voltage. However, the ${ }^{239} \mathrm{Pu}-\mathrm{Be}$ neutron source is also a source of gamma-rays, which might have affected the results.

In this paper we have examined SI GaAs detectors with a different type of neutron source to study their detection properties. The detectors were exposed to mono-energetic neutrons with higher kinetic energy (16.8 MeV) generated by a deuterium (D)-tritium (T) nuclear reaction at a Van de Graaff (VDG) accelerator. The detection properties were evaluated considering two parameters: the thickness of the HDPE conversion layer (varying from $50 \mu \mathrm{m}$ to $1300 \mu \mathrm{m}$ ) and the thickness of the active detector volume (varying from 60 up to $234 \mu \mathrm{m}$ ) modified by the detector reverse voltage (50 to $300 \mathrm{~V}$ ). 


\section{Detector Structure}

The neutron detectors were prepared from a bulk LEC (Liquid Encapsulated Czochralski) SI GaAs substrate made at CMK Ltd (Slovakia). The resistivity of GaAs is about $1.1 \times 10^{8} \Omega \mathrm{cm}$, and the Hall mobility is $5280 \mathrm{~cm}^{2} / \mathrm{Vs}$ at room temperature. The wafer was polished from both sides to a thickness of $270 \mu \mathrm{m}$. The Schottky electrode was evaporated onto the topside using $120 \mathrm{~nm}$ thick AuZn metallization. Its multi-pixel structure was created by a system of $8 \times 3$ large pixels $(400 \mu \mathrm{m} \times 400 \mu \mathrm{m})$ connected with $7 \times 2$ small pixels $(200 \mu \mathrm{m} \times 200 \mu \mathrm{m})$, as seen in Fig. 1, covering an area of $7.36 \mathrm{~mm}^{2}$. A rectangular quasi-ohmic metal electrode was formed by evaporating a eutectic AuGeNi alloy $(120 \mathrm{~nm})$ on the back side of the substrate.

The Schottky contact was covered by an HDPE conversion layer, where neutrons transfer their kinetic energy to protons in the hydrogen atoms through elastic nuclear collisions, as depicted in Fig. 2. The HDPE layer of 50, 650 or $1300 \mu \mathrm{m}$ thickness was fixed at the surface of the Schottky metallization by a dielectric polypropylene (PP) based glue (about $2 \mu \mathrm{l}$ ) diluted with toluene (10\% solution), minimizing the prospective roughness of the detector or HDPE layer and preventing any air gap formation. Moreover, the insulating nature of the PP glue decreases the detector reverse current by a few nA. ${ }^{7}$

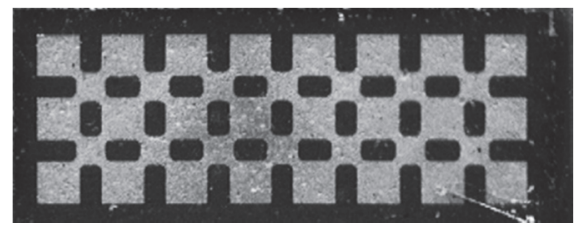

Fig. 1. Photograph of the multi-pixel structure of the detector Schottky contact.

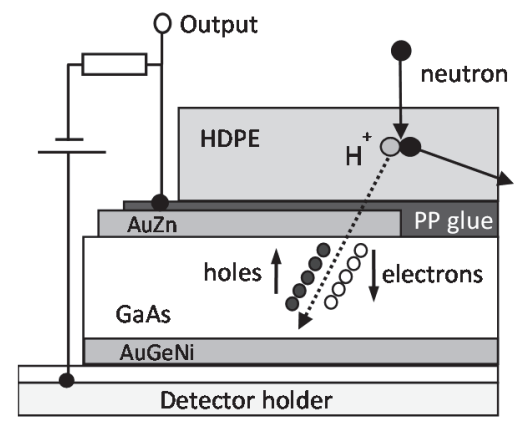

Fig. 2. Cross-section view of the SI GaAs detector with HDPE conversion layer for neutrons fixed by PP glue. 


\section{Experiment}

The measurements were performed at the Van de Graaff Laboratory of the Institute of Experimental and Applied Physics at the Czech Technical University in Prague. Fast neutrons were produced by bombarding a tritium target with deuterons accelerated by the VDG accelerator to $1 \mathrm{MeV}$ kinetic energy. The kinetic energy of the produced fast neutrons was $16.755 \mathrm{MeV}$ at 0 degree angle, ${ }^{9}$ and their flux on the sample was about $2.5 \times 10^{6} \mathrm{n} / \mathrm{cm}^{2} \mathrm{~s}$. The distance between the detector and the neutron source was about $3 \mathrm{~cm}$. The detector tested was connected to a spectrometric chain based on InSpector2000 readout electronics. The fast neutrons transfer their energy to hydrogen nuclei in HDPE by elastic scattering. The SI GaAs detector detects the recoil protons, ${ }^{5}$ and the HDPE layer is directly sensitive to the fast neutrons. ${ }^{10,11}$ As the protons are slowed down in the detector volume, electron-hole pairs are created. The high electric field between the detector electrodes separates the generated charges, and the pulse corresponding to proton energy can be measured at the output of the detector (see Fig. 2). The measured pulse-height spectra showing the influence of the HDPE thickness on the detection of neutrons are shown in Fig. 3. The effect of the active detector thickness determined by the applied reverse voltage can be seen in Fig. 4. The first steeply decreasing part of the spectra (up to channel 30) corresponds to the preamplifier and detector noise, and the second part (channels 30 up to 300 ) represents the neutron detection.

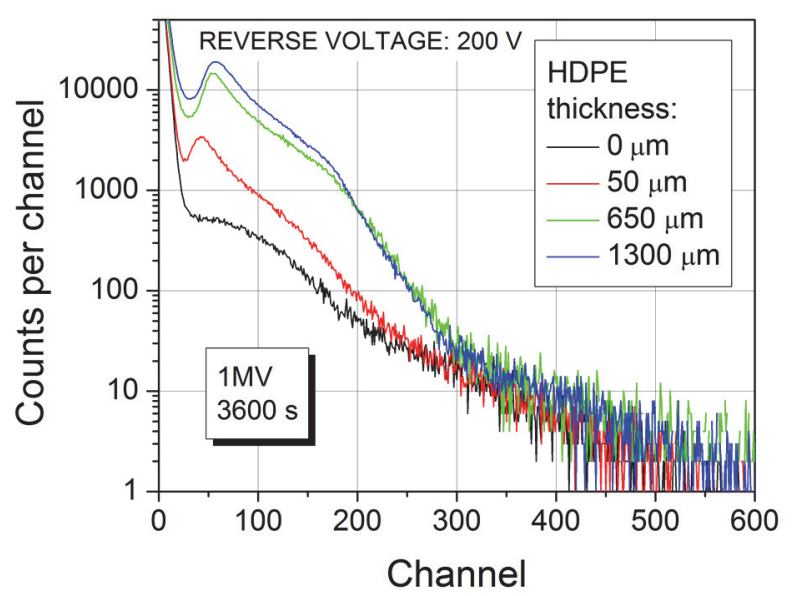

Fig. 3. Spectra of fast neutrons measured by SI GaAs detectors coated by an HDPE conversion layer of various thicknesses at a reverse voltage of $200 \mathrm{~V}$. 




Fig. 4. Spectra of fast neutrons measured by SI GaAs detectors coated by a $650 \mu \mathrm{m}$ thick HDPE conversion layer at different reverse voltages.

\section{Results and Discussion}

Two parameters were observed in our experiments: the neutron conversion layer thickness and the reverse voltage applied to the SI GaAs detector. The influence of these two parameters on the detection efficiency of neutrons was studied.

\subsection{Conversion layer thickness effect}

The HDPE conversion layer acts as a recoil proton source, and its thickness is an important parameter. We used HDPE layers of various thicknesses (50, 650 and $1300 \mu \mathrm{m})$ and compared the results with a bare detector. The conversion efficiency of the HDPE conversion layer is determined by two phenomena. The first is the number of neutrons recoiling the protons, which increases with the growing layer thickness. The second is the projected range of recoiled protons in the layer. The projected range of protons in HDPE increases with the proton energy, as is seen in Fig. 5. The maximum energy of recoiled protons in our experiment was $16.755 \mathrm{MeV}$, which means the maximum projected range is $\sim 3 \mathrm{~mm}$ according to SRIM calculations. ${ }^{12}$ If the conversion layer thickness exceeds the projected range of protons, some of them are absorbed in the layer and will not reach the active detector volume. The theoretical optimum thickness of the conversion layer can be determined by the Monte Carlo radiation transport computer code MCNPX. ${ }^{13}$ As shown in Ref. 14, the optimum values of the HDPE thickness, reaching the highest conversion efficiency, are shorter than the proton ranges in the layer calculated using the SRIM programme. ${ }^{12}$ The neutron conversion efficiency is expressed as a ratio of the number of recoiled secondary particles (protons) and the number of neutrons impinged on the detector surface. The conversion efficiency of the HDPE reactive layer steeply increases with its thickness up to an optimum value of about $1.9 \mathrm{~mm}$ for $16.5 \mathrm{MeV}$ neutrons, followed by a milder decrease. Because the thickness of 


\section{A. Sagatova et al.}

our HDPE layers ranges from $50 \mu \mathrm{m}$ up to $1300 \mu \mathrm{m}$, the neutron conversion efficiency increases with growing thickness, leading to a higher neutron detection efficiency of the SI GaAs detector, as shown in Fig. 6. A similar tendency with the conversion layer thickness was observed in Ref. 5, where the GaAs detector was coated by 125 and $875 \mu \mathrm{m}$ thick HDPE layers, detecting $14 \mathrm{MeV}$ neutrons.

The neutron detection efficiency $\varepsilon$ can be expressed as:

$$
\varepsilon(\%)=\frac{N}{F \times A \times t} \times 100,
$$

where $N$ is the number of counts above the valley, $F$ is the neutron flux (neutrons $/ \mathrm{cm}^{2} \mathrm{~s}$ ) on the sample, $A$ is the detector area in $\mathrm{cm}^{2}$ and $t$ is the exposure time in seconds. The investigated SI GaAs detectors were exposed to fast neutrons delivered by the VDG accelerator. The average neutron flux on the sample was $2.5 \times 10^{6} \mathrm{n} / \mathrm{cm}^{2} \mathrm{~s}$ during irradiation, varying from $2.3 \times 10^{6} \mathrm{n} / \mathrm{cm}^{2} \mathrm{~s}$ when using the SI GaAs detector with a $1300 \mu \mathrm{m}$ HDPE layer up to $2.7 \times 10^{6} \mathrm{n} / \mathrm{cm}^{2} \mathrm{~s}$ when the SI GaAs detector with a $650 \mu \mathrm{m}$ HDPE layer was tested.

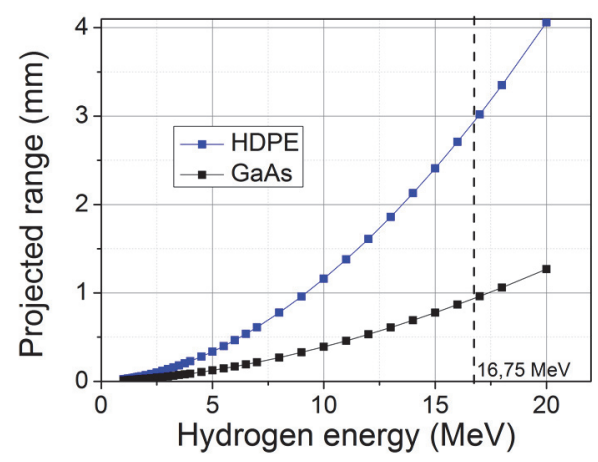

Fig. 5. Projected range of hydrogen nuclei recoiled by fast neutrons in the conversion layer (HDPE) vs. in detector material (GaAs) according to SRIM calculations. ${ }^{12}$

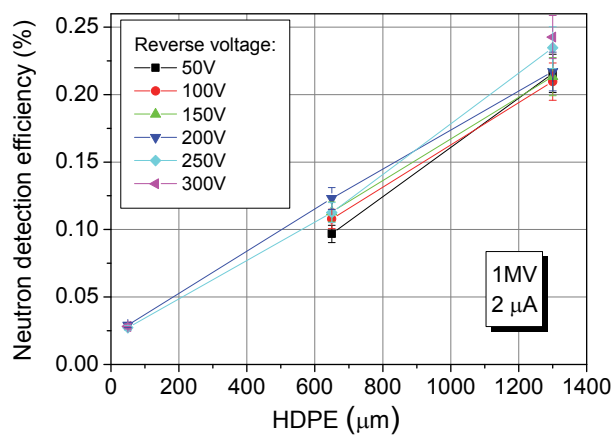

Fig. 6. Neutron detection efficiency of the SI GaAs detector coated by an HDPE neutron conversion layer as a function of the HDPE layer thickness for various reverse voltages applied. 


\subsection{Active detector thickness effect}

The knowledge of the electric field distribution across GaAs detectors is of major interest for their optimization. To improve the performance of the detector, a high and as extended electric field as possible is required. In this way we prevent trapping and can utilize nearly the whole detector as an active effective volume of detection. In Ref. 15 it was proved that for reverse voltages higher than $20 \mathrm{~V}$, the active layer thickness of the SI GaAs detector linearly increased with the voltage applied (see Table 1). By increasing the reverse voltage, we deepen the active detector volume, and a larger portion of the charge generated by slowing-down the protons is registered. It will be manifested by an increased number of counts in higher channels. At lower reverse voltage, the active detector volume is thinner, but the recoiled protons are registered alike. However, the smaller part of generated charge is collected. The pulse corresponding to the proton energy measured at the output of the detector is lower, thus registered in lower channels. We can follow this tendency in the spectra that were shown in Fig. 4.

Table 1. Thickness of the active volume of the SI $\mathrm{GaAs}$ detector as a function of the applied reverse voltage according to Ref. 15.

\begin{tabular}{cc}
\hline Reverse voltage $(\mathrm{V})$ & Active thickness $(\mu \mathrm{m})$ \\
\hline 50 & 59 \\
100 & 94 \\
150 & 129 \\
200 & 164 \\
250 & 199 \\
300 & 234 \\
\hline
\end{tabular}

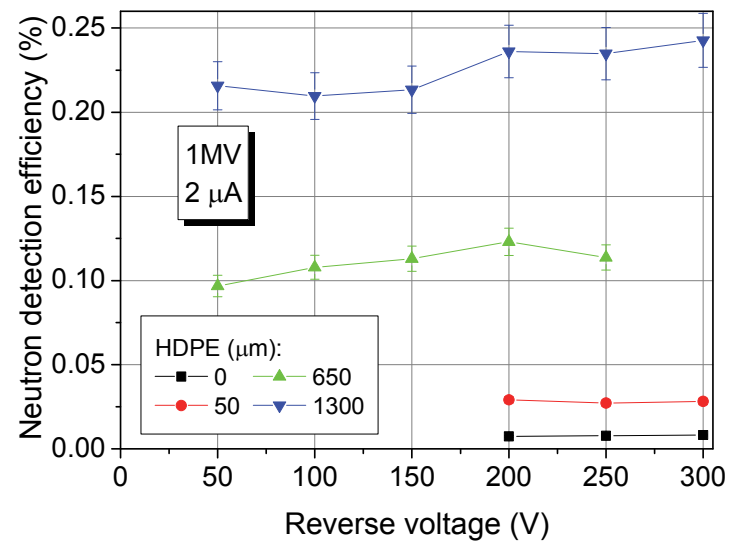

Fig. 7. Neutron detection efficiency of the SI GaAs detector coated by variously thick HDPE layers as a function of the reverse voltage applied. 
The count rate above the valley representing the detected protons should be constant and independent of the applied reverse voltage. Thus also the neutron detection efficiency should not change with voltage applied. However, our measurements showed a slight increase of detection efficiency with voltage at thicker HDPEs, which was most probably caused by the extending electric field. According to our previous results, ${ }^{16}$ the active volume of the SI GaAs detector expands not only to its depth but also to the sides, behind the Schottky contact edges. The multi-pixel structure of our Schottky contact will multiply this effect. This was the probable reason for the slight increase in neutron detection efficiency with increasing reverse voltage, observable with detectors with thicker HDPE layers $(650$ and $1300 \mu \mathrm{m})$ that exhibit a higher neutron conversion efficiency.

In our experiment, the maximum neutron detection efficiency of $0.243 \%$ was obtained by the detector with a $1300 \mu \mathrm{m}$ thick HDPE layer at $300 \mathrm{~V}$, which is in good agreement with other research groups dealing with semiconductor neutron detectors. In Ref. 4, the authors obtained a neutron detection efficiency of $0.13 \%$ using an epitaxial GaAs detector with a polyethylene converter exposed to an ${ }^{241} \mathrm{AmBe}$ neutron source (4.08 MeV mean neutron energy). Another group (Ref. 2) reached an intrinsic detection efficiency of $7.6 \times 10^{-4}$ using an ${ }^{241} \mathrm{AmBe}$ source and $2.5 \times 10^{-4}$ with ${ }^{252} \mathrm{Cf}(2.2 \mathrm{MeV}$ mean neutron energy) utilizing a semiconductor alpha detector with a polyethylene conversion layer. Research with a Medipix2-based silicon device coated by $1300 \mu \mathrm{m}$ of polyethylene showed a mean efficiency of $0.18 \%$ for $14 \mathrm{MeV}$ neutrons from a VDG (Ref. 3) and a neutron detection efficiency of about $0.09 \%$ for $4 \mathrm{MeV}$ neutrons from an ${ }^{241} \mathrm{AmBe}$ source. ${ }^{17}$ We have obtained an experimental detection efficiency of $0.113 \%$, using the optimal thickness of HDPE layer $(500 \mu \mathrm{m})$ for an ${ }^{241} \mathrm{AmBe}$ neutron source in our previous research described in Ref. 7. The detection efficiency could be affected by the detector and readout electronic noise, which superposed with signal from low energy protons.

\section{Conclusions}

The response of SI GaAs detectors to mono-energetic fast neutrons $(16.8 \mathrm{MeV})$ from the D-T nuclear reaction has been experimentally studied. First, the thickness of the HDPE neutron conversion layer and its influence on the neutron detection efficiency was the objective of the research. The HDPE thickness varied from 50 to $1300 \mu \mathrm{m}$. In this range, we observed an increasing neutron detection efficiency with greater HDPE thickness as the neutron conversion efficiency of the layer was also increasing. Second, the effect of active detector thickness modified by the detector reverse voltage was evaluated. By increasing the detector reverse voltage, the detector active volume expands to the depth of the GaAs substrate and also behind the edges of the blocking Schottky detector contact. The greater detector volume slightly increases the overall neutron detection efficiency in the case of thicker HDPE layers. 


\section{Acknowledgments}

This work was partially supported by the Slovak Grant Agency for Science through grants Nos. 2/0062/13, 2/0175/13, by the Slovak Research and Development Agency under contract No. APVV-0321-11 and by the Ministry of Education, Youth and Sports of the Czech Republic in the frame of the research funding grant MSMT No. LM2011030 "VdG Research Infrastructure".

\section{References}

1. A.N. Caruso, J. Phys. Condens. Matter 22, 443201 (2010).

2. $\quad$ R. Pollanen and T Siiskonen, Applied Radiation and Isotopes 90, 187 (2014).

3. D. Greiffenberg et al., Nucl. Instr. Meth. Phys. Res. A 607, 38 (2009).

4. A.V. Chernykh et al., JINST 10, C01021 (2015).

5. D.S. Mcgregor, R.T. Klann, H.K. Gersch, and Y.H. Yang, Nucl. Instr. Meth. Phys. Res. A 466, 126 (2001).

6. B. Zatko et al., JINST 6 C12047 (2011).

7. A. Sagatova et al., JINST 8 C03016 (2013).

8. $\quad$ P. Masek, J. Jakubek, J. Uher, and R. Preston, JINST 8 C01022 (2013).

9. H. Liskien and A. Paulsen, Nuclear Data Tables 11, 569 (1973).

10. H. Gotoh, H. Yagi, Nucl. Instr. Meth. 101, 395 (1972).

11. R.T. Klann and D.S. McGregor, in Conference Record of ICONE-8 (Eighth International Conference on Nuclear Engineering), No. 8110, (USA, MD, Baltimore, April 2-6, 2000).

12. J.F. Ziegler, J.P. Biersack, and M.D. Ziegler, SRIM - The Stopping and Range of Ions in Matter (Lulu Press Co., NC, USA, 2008), pp. 1-398.

13. D.B. Pelowitz (Ed.), MCNPX User's Manual Version 2.7.0 (Los Alamos National Laboratory Report, LA-CP-11-00438, April 2011), pp. 1-645.

14. K. Sedlackova et al., MCNPX simulations of the silicon carbide semiconductor detector response to fast neutrons from D-T nuclear reaction, in International Journal of Modern Physics: Conference Series, this volume.

15. A. Castaldini et al., Nucl. Instr. Meth. Phys. Res. A 410, 79 (1998).

16. A. Sagatova-Perdochova et al., Nucl. Instr. Meth. Phys. Res. A 563, 187 (2006).

17. J. Bouchami et al., Nucl. Instr. Meth. Phys. Res. A 663, 226 (2011). 